\section{THE SUCCESSFUL JOURNEY OF THE FIRST A2LA ISO 15189:2012 MEDICAL LABORATORY IN THE KINGDOM OF SAUDI ARABIA} (RCHSPY)

Ammar Ashari, Mahmoud Radwhan, Fuad Alshoabi. Planning Section/Quality Management and Planning Department, Royal Commission Health Services Program

\subsection{6/bmjoq-2019-PSF.22}

Background The planning-execution gap is one of the main challenges facing any organization. According to the literature, the planning- execution gap is one of the leading causes of strategic and operational plan failure. Because of the complexity of healthcare, planning is more challenging. Inspired by Saudi Vision 2030 and guided by the National Transformation Program 2020, Royal Commission Health Services Program Yanbu (RCHSPY) made its Strategic and Operational Plan 2017-2020. The planning section identified the planning-execution gap as one of the main challenges impeding the progress of RCHSPY plans. This gap can result from many causes, such as ineffective communication, lack of measures and feedback, lack of collaboration, and weak alignment between strategic and operational plans. The planning section tried to study the gap by assessing the existing status and identifying the root causes in RCHSPY, then applying several tools and techniques in order to overcome this gap.

Methods RCHSPY aims to evaluate the involvement and awareness of all staff, particularly front liners and junior staff, about strategic and operational plans to ensure that they are fully oriented about the direction of the RCHSPY and that they feel their daily work is aligned with the direction of the RCHPSY strategic and operational plan. Surveys were carried out to assess the planning effectiveness and the planning-execution gap. The surveys asked staff about orientation, active participation, alignment, collaboration, and understanding of the initiatives and key performance indicators (KPIs). The survey results were supported by the analysis of 2017 and 2018 operational plan outcomes, as some significant findings have been observed.

Results The survey results showed that $50 \%$ of staff feel unsatisfied with their orientation, 34\% feel unsatisfied about their participation in RCHSPY plans, 27\% feel that their daily work is not aligned with RCHPSY plans, 50\% feel unsatisfied about collaboration with other departments to implement different initiatives, and 25\% replied that they never discussed RCHSPY in their departments. The 2017 and 2018 operational plan analysis of stumbling projects and initiatives showed four main challenges $(4 \mathrm{C})$ : communication, collaboration, financial and managerial constraints, and connecting departmental KPIs to RCHSPY plans, which support the survey results.

Conclusion More effective communication methods are greatly required, such as interactive workshops and face-toface meetings, to enhance the involvement and empowerment of all staff in the planning process. Feedback should be delivered to all concerned stakeholders in the form of quarterly reports and KPI results should be discussed at all levels, particularly at the departmental level. Future studies about the planning-execution gap should be done to monitor progress.
Kaneez Zamir, Omar Qassas, Abdulaziz Johani, Abdulrahman Aboud. Department of Pathology and Laboratory Medicine, Prince Mohammed Bin Abdul Aziz Hospital - Al Madinah

\subsection{6/bmjoq-2019-PSF.23}

Background The aim was to improve the overall quality management system for the Department of Pathology and Laboratory Medicine by attaining international accreditation to A2LA ISO 15189 , in order to provide accurate, reliable, and timely results for excellent patient care and patient safety. The accreditation of laboratory medicine improves all processes in the laboratory, including reduction of errors in the pre-analytical, analytical, and post-analytical processes, facilitation of accurate and rapid diagnostics, participation in acceleration and efficiency of treatment, and stimulation of continuous improvement; therefore, the decision was taken to aim for A2LA ISO 15189 accreditation.

Methods

- Baseline assessment of the serology section was chosen as the model of implementation once successfully completing of all the identified gaps from the ISO 15189 standard.

- Development of project management action plan and agreed upon timeline:

- Accreditation Project Initiation 2015

- Accreditation Project Identification and Planning 2015-2016

- Accreditation Project Execution 2016-2017

- The ISO 15189 parameters were applied to the serology section and monitored by use of key performance indicators (KPIs).

- Accreditation Project control 2017

- The control was through performing internal audits and then taking corrective action with preventive action.

- Accreditation Project Closeout 2018

- The project was submitted for application to the American Association for Laboratory Accreditation ISO 15189.

Results Internal audits were performed from the baseline audit in 2015 to the final exit audit in 2018. The results for all the sections were as follows:

Chemistry: 2015 - 20\%; 2016 - 40\%; 2017 - 46\%; 2018 - 95\%. Hematology: 2015 - 51\%; 2016 - 60\%; 2017 - 62\%; $2018-91 \%$. Microbiology: 2015 - 19\%; 2016 - 40\%; 2017 - 46\%; 2018 - 85\%. Serology: 2015 - 52\%; 2016 - 65\%; 2017 - 83\%; 2018 - 95\%. Receiving OPD: 2015 - 39\%; 2016 - 52\%; 2017 - 70\%; 2018 - 90\%. Blood bank: 2015 - 20\%; 2016 - 50\%; 2017 - 70\%; 2018 - 83\%. Molecular biology: 2016 - 20\%; 2017 - 60\%; $2018-85 \%$.

Histopathology: 2015 - 15\%; 2016 - 30\%; 2017 - 60\%; 2018 - 89\%.

From the results, between 2016 and 2018, a dramatic increase in compliance was demonstrated, and therefore preparation for the final A2LA ISO 15189 audit was executed.

Conclusion An essential component of successful implementation of A2LA ISO 15189 was the coordination and rapport between management and staff. Well-trained and well-motivated laboratory staff were required to implement the system. The initial gap analysis and then the continuous monitoring through internal and external assessment provided invaluable 
tools for the successful accreditation to ISO 15189:2012 for more than 250 tests. The Department of Pathology and Laboratory Medicine was the first laboratory in KSA to gain the A2LA ISO 15189 accreditation.

\section{DEVELOPMENT OF AN ANTIMICROBIAL STEWARDSHIP EDUCATIONAL CURRICULUM FOR NURSES: THE LEARNING NEEDS ASSESSMENT}

Nour Shamas, Elham Bukhari, Asim Al Saedi, Wesam Talal Abuznadah. Infection Prevention and Control King Abdulaziz Medical City - WR

\subsection{6/bmjoq-2019-PSF.24}

Background The Antimicrobial Stewardship Program (ASP) at King Abdulaziz Medical City - Western Region (KAMC-WR) aims to improve antimicrobial use through several interventions including education of healthcare providers. Nursing represents more than a third of the health workforce at KAMCWR. Nurses' important role in facilitating the implementation of ASP has been recognized and is supported by the literature. In developing a customized educational curriculum to reinforce the role of nursing in antimicrobial stewardship (AS), this study has been done, in collaboration with the Nursing Education Department, to assess nurses' learning needs. Although studies have examined AS knowledge, there is no empirical data regarding assessment of nurses' learning needs of AS in Saudi Arabia.

Methods A descriptive, cross-sectional survey of all levels of nursing staff using a learning needs assessment tool was implemented. The tool consisted of 13 questions aiming to assess nurses' perceived knowledge of antimicrobials' spectrum of activity, usage, indication, duration, adverse effects, antimicrobial resistance, Clostridium difficile infection, vaccination, antibiotic prophylaxis, and questions related to diagnostic modalities for infectious syndromes. A five-point Likert scale was used with responses ranging from 'satisfied' to 'dissatisfied' and 'not applicable'. Questionnaires were validated by circulating the survey to ten nurses with a varying number of years of experience, and assessing their responses. Validated questionnaires were handed out by the nurse managers of all 31 NGHA departments. Simple descriptive statistics were used for data analysis.

Results The response rate was 43\% (608 of 1411). Cardiac, oncology, and surgery departments represented most of the responses. Nurses' years of experience ranged from 0 to 32 years. The majority of nurses were SN1 and 2 (56\% and $32 \%$, respectively). Knowledge was mainly lacking in identification of patients with possible $\mathrm{C}$ difficile infection, prolonged antimicrobial infusion protocols, and rapid diagnostic test sampling techniques. 'Neutral' was the mode for most answers of 8 of 13 questions, and the rest were 'moderately satisfied'. The 'not applicable' category was chosen by $2-5 \%$ of responders depending on the question. This indicates that many nurses are unsure of their need for training, and that some nurses do not even identify their crucial role in AS.

Conclusion In this study, nurses' learning needs of AS were assessed. The results show that nursing staff are in need of professional development and training on AS concepts and their key role in ASPs. The results of this survey will help the ASP at KAMC-WR build a tailored educational curriculum that aims to improve overall patient care.

\section{A QUALITY IMPROVEMENT PROJECT ON PATIENT SAFETY EDUCATION IN THE OUTPATIENT DEPARTMENT (OPD)}

Mohammad Faisal Salapuddin, Graceslick Atienza, Sitti Fatima Isnani, Mwanaid Binali. Outpatients Department, Prince Mohammed Bin Abdulaziz Hospital - Ministry of National Guard Health Affairs

\subsection{6/bmjoq-2019-PSF.25}

Background It is an ethical responsibility of registered nurses to provide health education to their patients as part of their nursing care. However, in reality, it is often not carried out because of several reasons. As a result, a quality improvement project was done in the OPD PMBAH-MNGHA. A quality team was created, which identified the absolute lack of patient education as evidenced by a survey on nurses' documentation from 1-15 February 2017. The survey showed that among 30 patients, zero patient education was provided by nurses during the period. Furthermore, a fishbone analysis showed two primary reasons for the lack of patient education: (1) unavailability of a standard patient education tool or guidelines for nurses; and (2) inadequate training support on the use of the hospital's intranet resources. Therefore, the objective of the project is to increase patient education in outpatients by at least $50 \%$ in 12 months.

Methods A PDSA (plan-do-study-act) cycle was used as a framework for developing, testing, and implementing changes for improvement. The team created a standard patient safety education guidelines tool, which contains seven topics about patient safety: medication safety, hand hygiene, fall prevention, proper patient identification, pain score tool, pressure ulcer prevention, and general control of infection. Furthermore, TeamStepps principles and strategies were used during the implementation. The outcome measure of the project was the total number of documented cases of patient education while process measures were staff documentation of patient education, staff training support, and use of patient education tool. A small test of change was done from 26-30 March 2017 prior to implementation. A simple random sampling method was used for data collection.

Results A small test of change gained a result of $80 \%$ compliance rate. From April to December 2017 compliance was $71 \%$ and in 2018 compliance was $84 \%$. The hospital's outpatient department has gone from zero patient education in February 2017 to a compliance rate of $84 \%$ in 2018 using the patient education guidelines tool as evidenced by nursing documentation.

Conclusion The project has established a quality improvement as part of ensuring the delivery of quality patient care to our clients and their families. Finally, registered nurses can perform their function as health educator to patients and their families as part of nursing care through the use of the patient safety education guidelines tool. The project can be further improved with observational study of actual patient education provided and patients' feedback. 